\title{
High expression of miR-15b predicts poor prognosis for hepatocellular carcinoma after curative hepatectomy
}

\author{
WEN-BIN JI ${ }^{*}$, XIN LIU*, YING LUO and WEN-ZHI ZHANG \\ Department of Hepatobiliary Surgery, Chinese PLA General Hospital, Chinese PLA Postgraduate Medical School, \\ Beijing 100853, P.R. China
}

Received November 21, 2015; Accepted December 27, 2015

DOI: $10.3892 /$ or.2016.4982

\begin{abstract}
The present study aimed to analyze the role and mechanism of miR-15b in hepatocellular carcinoma (HCC) after curative hepatectomy. Tissue samples from 13 patients with HCC who were operated on at the Chinese PLA General Hospital from March 2014 to May 2014 were collected. A consecutive 156 untreated patients with HCC who received curative hepatectomy at the Chinese PLA General Hospital (Beijing, China) from May 2008 to March 2009 were enrolled, and their corresponding para-tumoral and normal tissue samples were acquired. Subsequently, anti-miR-15b (inhibitor) was transfected into human HCC HepG 2 cells. It was observed that high expression of miR-15b promoted cell proliferation of the HCC cells, while low expression of miR-15b suppressed cell growth and induced the apoptosis of HepG2 cells. It was found that overall survival of the patients with low miR-15b was increased, compared with the overall survival of the patients with high miR-15b expression. In addition, low expression of miR-15b suppressed the growth of HepG 2 cells by suppression of transforming growth factor- $\beta$ (TGF- $\beta$ ), T $\beta$ RI and Smad2 protein expression. Meanwhile, low expression of miR-15b significantly activated Bax protein expression and caspase- 3 activity in the HepG2 cells. The study results revealed that high expression of miR-15b could predict the poor prognosis of hepatocellular carcinoma after curative hepatectomy through TGF- $\beta$ /T $\beta$ RI-Smad2-cyclin D1/Bax.
\end{abstract}

Correspondence to: Dr Wen-Zhi Zhang, Department of Hepatobiliary Surgery, Chinese PLA General Hospital, Chinese PLA Postgraduate Medical School, 28 Fuxing Road, Haidian, Beijing 100853, P.R. China

E-mail: lue082966@126.com

*Contributed equally

Key words: miR-15b, hepatocellular carcinoma, TGF- $\beta$, Smad2, cyclin D1, Bax

\section{Introduction}

Hepatocellular carcinoma (HCC) is a common malignant cancer worldwide and is diagnosed in an increasing number of individuals (1). According to the data since $2000, \mathrm{HCC}$ is the fifth most common malignant cancer among males and the eighth most common cancer among females (2). As a probable radical cure, surgical resection remains the optimal method for patients in China and many other countries (3). Through regular application of ultrasound screening for patients suffering liver cirrhosis or hepatitis, many patients are diagnosted who are suitable for hepatectomy. With the development of surgical techniques and perioperative management, hepatectomy has become a secure method for HCC with rare complications (4). In the past 30 years, the survival rate of HCC patients after resective surgery has been significantly improved. However, in China and Southeast Asia, as HCC is secondary to viral hepatitis and liver cirrhosis, postoperative recurrence is the main cause of treatment failure (5).

$\mathrm{HCC}$ is a disease with a high incidence in China. Hepatic fibrosis refers to a disease characterized by over-deposition of extracellular matrix (ECM) and hepatic function damage caused by various types of continuous hepatic trauma repair reacting to chronic diseases (6). TGF- $\beta$ is the most important cytokine in fibrosis, as it plays an essential role in promoting hepatic stellate cells (HSCs) to secrete collagens (6). TGF- $\beta$ is generally secreted in an inactive form. However, when under a stress state, the potential TGF- $\beta 1$ compound will be activated by a tissue damage-specific mechanism to exert its biological effects. As a messenger protein molecule after the specific receptor of the TGF- $\beta$ superfamily, Smad exerts intermediary functions by introducing cell nucleus from cytomembrane through mediating TGF- $\beta$ signals. The basic procedure of the TGF- $\beta /$ Smad signaling pathway expression is as follows. After stimulating factors which act on HSCs, the auto-phosphorylated T $\beta$ RII combines with the TGF $\beta$ ligand under the auxiliary function of T $\beta$ RIII to activate transmembrane T $\beta R$. Subsequently, after phosphorylation of transmembrane receptors, the Smad proteins combine with synergistic Smad of Smad4 to form compounds, which will be transmitted into the cell nucleus. Finally, the activated compounds combine with cofactors of two DNAs to determine the transcriptional activity of target genes. After entering the nucleus, Smad complexes combine with the specific sequence 
of the promoters of target genes to form stable compounds to regulate target genes indirectly. Meanwhile, Smad complexes combine directly with DNA in the cell nucleus to activate the transcription of target genes. After the activation of HSCs, an extensive amoount of ECM will be deposited in hepatic cells. Furthermore, the activated HSCs can also secrete TGF- $\beta$ to form cascade reactions, which promote the incidence of fibrosis (7).

Smurf2 can selectively act on R-Smad proteins. With the PPXY sequence, Smad2 and Smad3 can combine with Smurf 2 through WW structural domain. Without the prolinetyrosine PPXY sequence, Smad4 cannot be directly combined with Smad2, but can be regulated only through indirect pathways (8). Studies have shown that the Smad4 level cannot be changed when cells are separately transfected by Smad7 genes while they can only be downregulated when cells are independently transfected with Smads2. However, if cells are transfected by Smad7 and Smurf2 simultaneously, the expression of Smad4 will significantly decrease (9). In addition, it is observed that Smad7 can activate enzymatic activities through facilitating Smurf2 to gather E2, which plays multiple functions in regulating TGF- $\beta$ pathways (8).

Playing an essential role in HCC, miRNAs directly participate in proliferation, apoptosis, differentiation, invasion and metastasis of HCC cells (10). Studies also verify that the expression profiles of miRNAs are closely associated with the pathological features of HCC such as pathological patterns, stage and grades of malignancy (11), indicating that miRNAs can be utilized for not only diagnosis and individualized treatment but also as a technique to assess the prognosis of HCC (12). Therefore, identification of the miRNAs which play a vital role in HCC development and its function is expected to provide a new approach for HCC diagnosis and treatment. Notably, miR-15b is located on sites of genomes related with blood pressure, diabetes and prostatic cancer, suggesting that miR-15b may participate in regulating the processes of these diseases (13). miR-15b and miR-16 have various types of target genes, including proteins related to cell proliferation, cell cycle and apoptosis, such as cyclin D1, cyclin D3, cyclin El and CDK6 which are associated with cell cycle regulation. These proteins play crucial roles in cell cycle progression from G1 stage to other stages. In the present study, we demonstrated that the high expression of miR- $15 b$ is a predictor of the poor prognosis of HCC after curative hepatectomy.

\section{Materials and methods}

Patients and clinical specimens. All HCCs in the patients in this study were confirmed by histological diagnosis. Consent from all patients and approval from the Ethics Committee of the Chinese PLA General Hospital (Beijing, China) were obtained. Thirteen patients with HCC diagnosed from March 2014 and May 2014 at the Chinese PLA General Hospital were enrolled. Their tumor, para-tumor $(\leq 2.0 \mathrm{~cm}$ distance from the tumor edge) and normal $(>2.0 \mathrm{~cm}$ distance from the tumor edge) tissue samples were acquired after patient consent. A consecutive 156 untreated patients with HCC who received curative hepatectomy were enrolled from May 2008 to March 2009 at the Chinese PLA General Hospital. Curative hepatectomy conformed to the following criteria: cancer tissue
Table I. Correlations between miR-15b expression and clinicopathological characteristics.

\begin{tabular}{|c|c|c|c|}
\hline & $\begin{array}{l}\text { High miR-15b } \\
\qquad(\mathrm{n}=65)\end{array}$ & $\begin{array}{l}\text { Low miR-15b } \\
\quad(n=91)\end{array}$ & P-value \\
\hline \multicolumn{4}{|l|}{ Gender } \\
\hline Male & 57 & 78 & 0.713 \\
\hline Female & 8 & 13 & \\
\hline Age (years) & $46.2 \pm 11.1$ & $45.9 \pm 12.6$ & 0.581 \\
\hline$\leq 55$ & 55 & 74 & \\
\hline$>55$ & 10 & 17 & \\
\hline AFP level (ng/ml) & & & 0.974 \\
\hline$\leq 400$ & 33 & 48 & \\
\hline$>400$ & 32 & 43 & \\
\hline Tumor size $(\mathrm{cm})$ & $5.84 \pm 2.9$ & $6.41 \pm 3.1$ & 0.069 \\
\hline$\leq 5$ & 34 & 47 & \\
\hline$>5$ & 31 & 44 & \\
\hline Tumor encapsulation & & & 0.809 \\
\hline Complete & 33 & 31 & \\
\hline Incomplete & 32 & 60 & \\
\hline TNM stage & & & 0.029 \\
\hline I & 29 & 42 & \\
\hline II-III & 36 & 49 & \\
\hline
\end{tabular}

Table II. Primers for miR-15b, TGF- $\beta$ and Smad2.

\begin{tabular}{ll}
\hline Gene & \multicolumn{1}{c}{ Primers } \\
\hline miR-15b & F: 5'-GCGAGCACAGAATTAATACGACTCAC-3' \\
miR-15b & R:5'-GCGAGCACAGAATTAATACGACTCACTATAGG-3' \\
U6 & F: 5'-GCTTGCTTCGGCAGCACATATAC-3' \\
U6 & R: 5'-TGCATGTCATCCTTGCTCAGGG-3' \\
\hline
\end{tabular}

sample $\leq 3$, no vascular and bile duct invasion; microscopically complete removal of cancers; and no lymph node or distant metastasis. The main clinical and pathological variables of all patients are described in detail in Table I.

Reverse transcription-polymerase chain reaction (RT-PCR). Total RNAs were extracted from different tissue samples using TRIzol reagent (Invitrogen, Carlsbad, CA, USA) according to the manufacturer's protocol. Five micrograms of total RNA was transcripted with DNase I (Promega) for $15 \mathrm{~min}$ at room temperature and then polyA using polymerase (NEB, Ipswich, MA, USA) was added at $37^{\circ} \mathrm{C}$ for $1 \mathrm{~h}$. Total RNA (1.0 g) was transcripted with River-Tra Ace (Toyobo, Tokyo, Japan), oligo(dT) ${ }_{20}$, RNase inhibitor, 5-RT buffer, and dNTP mixture. RT-PCR was performed at $42^{\circ} \mathrm{C}$ for $20 \mathrm{~min}$ and then at $95^{\circ} \mathrm{C}$ for $5 \mathrm{~min}$ using $1.0 \mathrm{~g}$ of RNA per reaction. The ABI 7300 HT sequence detection system (Applied Biosystems, Foster City, CA, USA) was used to execute RT-PCR to detect the expression of miR-15b in all tissue and cell samples. The primers of miR-15b are listed in Table II. The quantitative miR-15b, TGF- $\beta$ and Smad2 expression was determined using the $2^{-\Delta \Delta \mathrm{Ct}}$ method. 


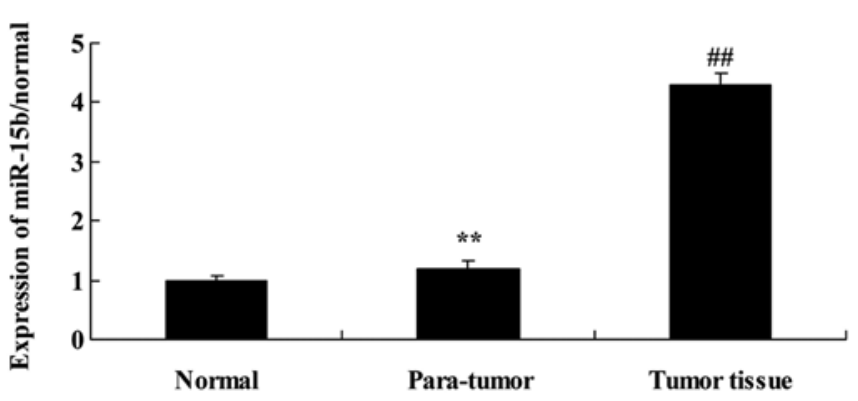

Figure 1. RT-PCR analysis of miR-15b expression in the normal, para-tumor and tumor tissues. ${ }^{* *} \mathrm{P}<0.01$ compared with the normal group; ${ }^{\# \prime \prime} \mathrm{P}<0.01 \mathrm{com}-$ pared with the para-tumor tissue group.

Immunohistochemistry. The tissue samples from the tumor and para-tumor samples were fixed with formalin, embedded in paraffin, cut into $4-\mu \mathrm{m}$ thick sections, de-waxed in xylene and rehydrated with graded concentrations of ethanol. The sections were blocked with $0.3 \%$ hydrogen peroxide, and then incubated with antigens in a microwave oven in $10 \mathrm{mM}$ citrate buffer for $30 \mathrm{~min}$. Next, the sections were washed three times using PBS and then incubated with anti-TGF- $\beta$ (1:500) and Smad2 (1:500) (both from Abcam, Cambridge, MA, USA) overnight at $4^{\circ} \mathrm{C}$. The sections were incubated with horseradish peroxidase-conjugated secondary antibody and developed with diaminobenzidine tetrahydrochloride (DAB; Beyotime Institute of Biotechnology, Jiangsu, China) and counterstained with hematoxylin.

Follow-up. In the first 2 years after surgery, follow-up of the patients included physical examination and routine laboratory testing every 3 months. Following 3 to 5 years after surgery, follow-up of the patients included a physical examination and routine laboratory testing every 6 months.

Cell culture and cell transfection. Human hepatocyte cell line MIHA and human HCC cell lines HepG2, PLC and 97H were cultured in Dulbecco's modified Eagle's medium (DMEM; Hyclone, Logan, UT, USA) supplemented with $10 \%$ fetal bovine serum (FBS; Gibco, USA) at $37^{\circ} \mathrm{C}$ in a $5 \% \mathrm{CO}_{2}$ incubator. The oligonucleotides of anti-miR-15b (inhibitor) were synthesized by GenePharma (Shanghai, China) bases with the following sequences: sense, 5'-UUCUCCGAACGUGUC ACGUTT-3' and antisense, 5'-ACGUGACACGUUCGGA GAATT-3'. HepG2 cells $\left(2 \times 10^{5}\right)$ were placed into each well of 6 -well plates for $24 \mathrm{~h}$. Anti-miR-15b (inhibitor) was transfected with Lipofectamine 2000 (Invitrogen) according to the manufacturer's instructions and incubated for $8 \mathrm{~h}$ before a change in medium.

MTT assay. The transfected HepG2 cells were seeded into 96-well plates at $3 \times 10^{3}$ cells/well. Fifty microliters of 3-(4, 5-dimethylthiazol-2-yl)-2,5-diphenyltetrazolium bromide (MTT) dilution ( $5 \mathrm{mg} / \mathrm{ml}$; KeyGen, Jiangsu, China) was added into each well for an additional $4 \mathrm{~h}$. The supernatant was removed and $200 \mu 1$ of DMSO (Invitrogen) was added to each well to dissolve the precipitate. The cell growth of the HepG2 cells was measured at a wavelength of $570 \mathrm{~nm}$.
Apoptosis assay. The transfected HepG2 cells were seeded into 6-well plates at $1 \times 10^{6}$ cells/well. Ten microliters of Annexin V FITC and $5 \mu \mathrm{l}$ PI (both from KeyGen) were used to double-stain the HepG2 cells in the dark for $30 \mathrm{~min}$ at room temperature. Then, apoptosis was detected and quantified by flow cytometry (Becton-Dickinson, USA).

ELISA analysis of caspase-3 activity. The transfected HepG2 cells were seeded into 6 -well plates at $1 \times 10^{6}$ cells/well. Total proteins were extracted and the protein concentration was determined by the BSA method (KeyGen). Fifty micrograms of total proteins were incubated with DEVD-pNA at $37^{\circ} \mathrm{C}$ for $30 \mathrm{~min}$. Caspase-3 activity was measured at a wavelength of $405 \mathrm{~nm}$.

Western blot analysis. The transfected HepG2 cells were seeded into 6 -well plates at $1 \times 10^{6}$ cells/well. Total proteins were extracted and the protein concentration was determined by the BSA method (KeyGen). Fifty micrograms of total proteins were subjected to SDS-PAGE on 10-12\% acrylamide gel and transferred to $\mathrm{NC}$ membranes (Millipore Corporation, USA). The membrane was blocked in 5\% nonfat milk in TBST (10 mM Tris- $\mathrm{HCl}$ buffer, $\mathrm{pH} 8.0,150 \mathrm{mM} \mathrm{NaCl}, 0.1 \%$ Tween-20) and incubated with diluted antibodies against TGF- $\beta(1: 2,000)$, T $\beta$ RI $(1: 3,000)$, Smad2 $(1: 4,000)$, cyclin D1 $(1: 4,000)$, Bax $(1: 4,000)$ and $\beta$-actin $(1: 5,000)$ (all from Santa Cruz Biotechnology, USA) overnight at $4^{\circ} \mathrm{C}$ followed by incubation with HRP-conjugated secondary antibody $(1: 3,000$; Santa Cruz Biotechnology).

Statistical analysis. Statistical analyses were performed using SPSS 17.0 statistical software (SPSS Company, Chicago, IL, USA). The correlation between the expression of miR- $15 \mathrm{~b}$ and clinicopathological characteristics of the HCC patients were analyzed with the Chi-square test. Results are expressed as mean \pm SD. Differences with $\mathrm{P}<0.05$ were considered statistically significant.

\section{Results}

RT-PCR analysis of miR-15b expression in normal, para-tumor and tumor tissues. To explore miR-15b expression in normal, para-tumor and tumor tissues, the expression of miR-15b was examined by RT-PCR analysis. As shown in Fig. 1, the expression of miR-15b in the para-tumor tissue samples was higher than that in the normal tissue samples. The results of RT-PCR analysis also revealed that the expression of miR-15b in the tumor tissue samples was higher than that in the para-tumor tissue samples (Fig. 1).

Immunohistochemical analysis of TGF- $\beta$ in the tumor and para-tumor tissues. To determine the biological functions of TGF- $\beta$ in the tumor and para-tumor tissues, we used immunohistochemical analysis of TGF- $\beta$ protein in the HCC patient tumor and para-tumor tissues. The results of the immunohistochemistry showed that TGF- $\beta$ protein in the para-tumor tissue samples was extremely lower than the level detected in the tissue samples (Fig. 2).

Immunohistochemical analysis of Smad2 in the tumor and para-tumor tissues. The impact of Smad2 was identified in the 


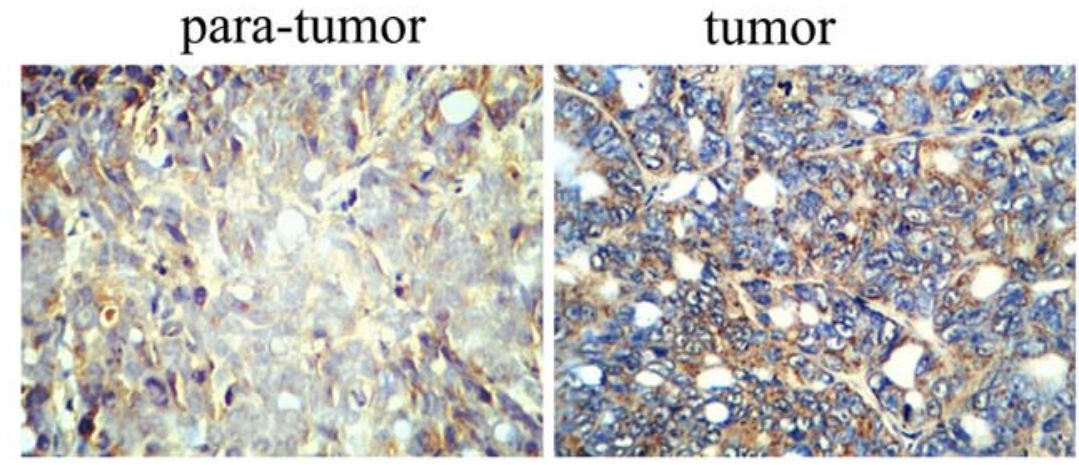

Figure 2. Immunohistochemical analysis of TGF- $\beta$ in para-tumor and tumor tissues (magnification, $\mathrm{x} 400$ ).

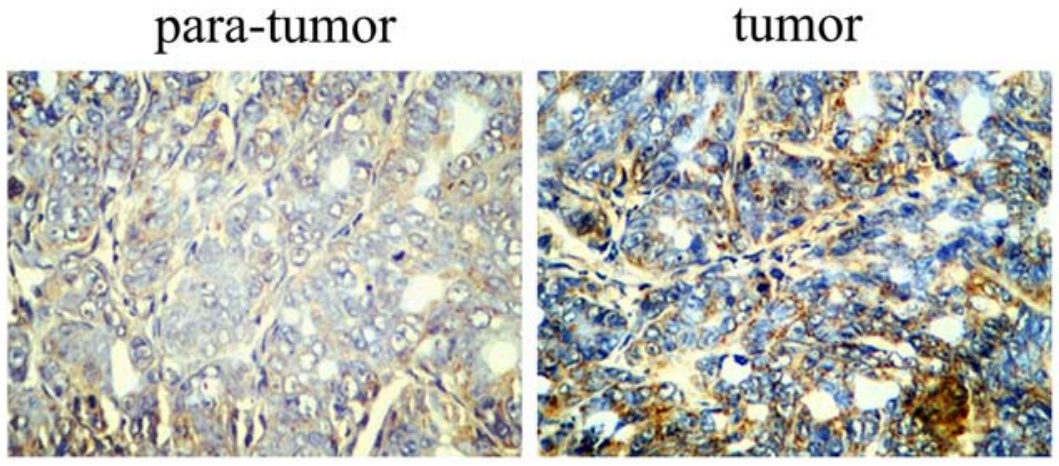

Figure 3. Immunohistochemical analysis of Smad2 in para-tumor and tumor tissues (magnification, $\mathrm{x} 400$ ).

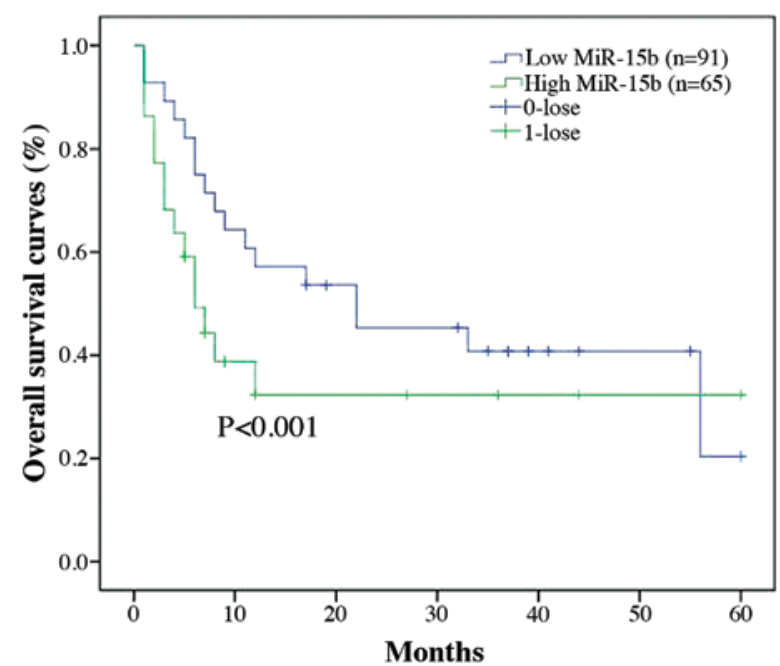

Figure 4. Overall survival curves of HCC patients after curative hepatectomy assessed according to miR-15b expression. ${ }^{* *} \mathrm{P}<0.001$ compared with the normal group.

tumor and para-tumor tissues. Immunohistochemical analysis was employed to determine the protein expression of Smad2 in the HCC tumor and para-tumor tissues. Fig. 3 shows that Smad2 protein expression was downregulated in the paratumor tissue samples, compared with the level detected in the tumor tissue samples.

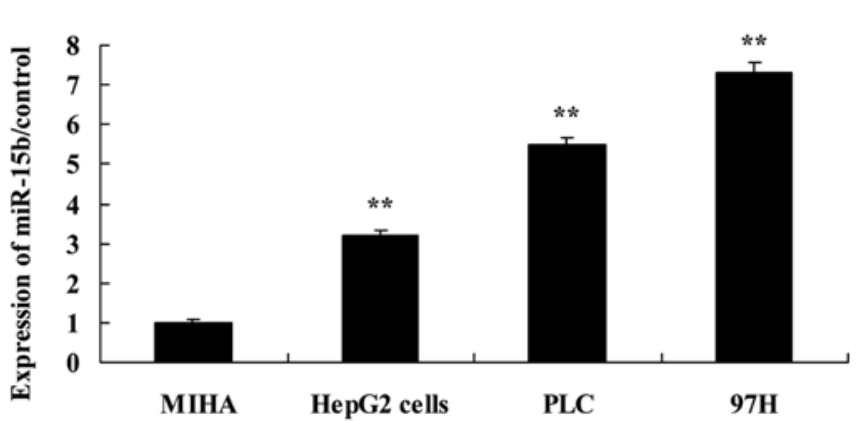

Figure 5. miR-15b expression in human hepatocyte cell line MIHA and human HCC cell lines HepG2, PLC and $97 \mathrm{H} .{ }^{* *} \mathrm{P}<0.01$ compared with the normal MIHA cell group.

Overall survival curves of HCC patients after curative hepatectomy assessed according to miR-15b expression. To investigate the association between miR-15b and overall survival (OS) of the HCC patients after curative hepatectomy, we analyzed the OS curves of HCC patients after curative hepatectomy as assessed by Kaplan-Meier analysis. As shown in Fig. 4, OS of the HCC patients with low miR-15b was increased, compared with the OS of the patients with high miR-15b.

miR-15b expression in the HCC cells. To investigate the expression of miR-15b in human hepatocyte cell lines MIHA and human hepatocellular carcinoma cell lines HepG2, PLC and 

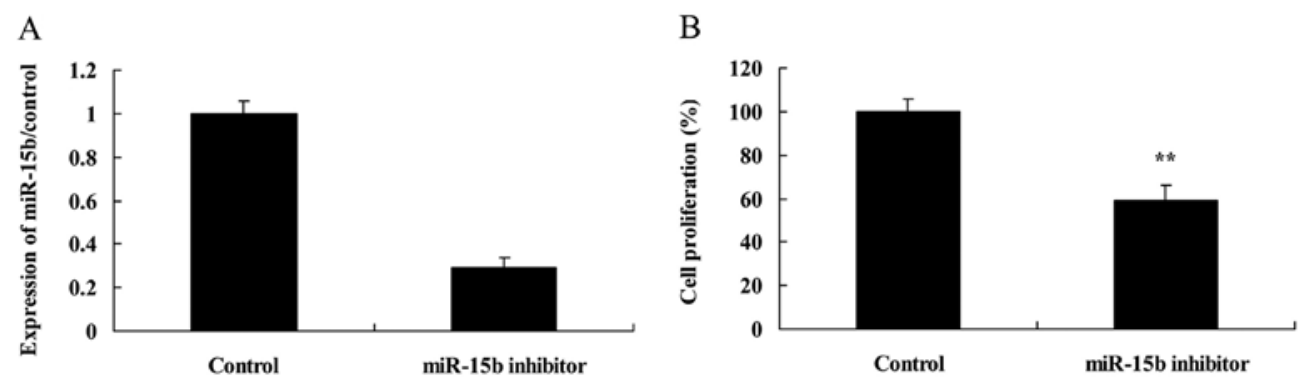

Figure 6. Anti-miR-15b (inhibitor) effectively diminished miR-15b expression (A) and suppressed the proliferation of HepG2 cells (B), compared with the control group. ${ }^{* *} \mathrm{P}<0.01$ compared with the control group.
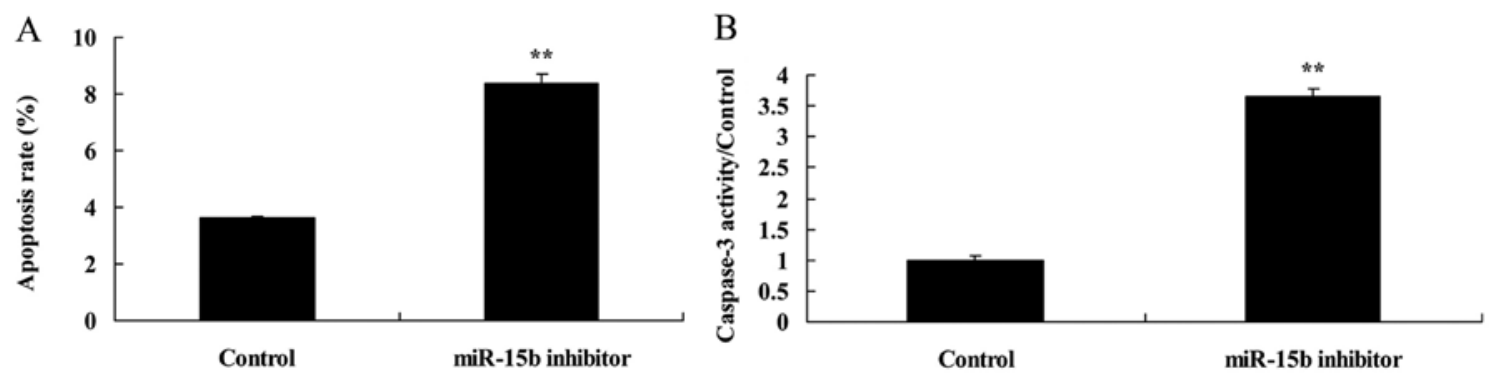

Figure 7. Effect of the downregulation of miR-15b expression on the apoptosis and caspase-3 level in the HepG2 cells. HepG2 cells transfected with the miR-15b inhibitor displayed an enhanced apoptosis rate (A) and caspase-3 activity (B) compared with the control group. ${ }^{* *} \mathrm{P}<0.01$ compared with the control group.

A

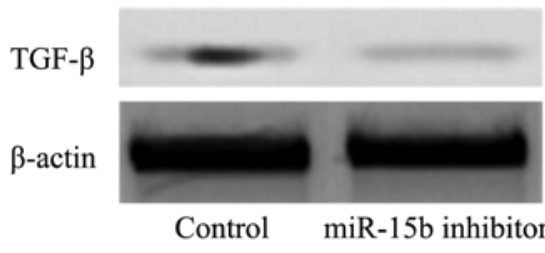

$\mathrm{B}$

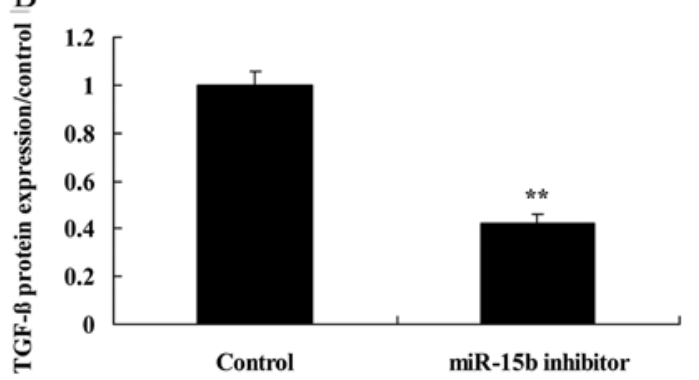

Figure 8. Downregulation of miR-15b expression reduced TGF- $\beta$ signaling in the HepG2 cells compared with the control group. Downregulation of miR-15b expression reduced TGF- $\beta$ protein expression using Western blot (A) and TGF- $\beta$ protein expression using statistical analysis (B) in the HepG2 cells compared with the control group. ${ }^{* *} \mathrm{P}<0.01$ compared with the control group.

97H cells, miR-15b expression was analyzed using RT-PCR analysis. As shown in Fig. 5, RT-PCR analysis revealed that expression of miR-15b in the HepG2, PLC and 97H cells was significantly activated, compared with the level in the MIHA cells.

Effect of the downregulation of miR-15b expression on the growth of HepG2 cells. To further examine the effect of miR-15b on the cell growth of HepG2 cells, cell proliferation of HepG2 cells was measured using the MTT assay. Importantly, anti-miR-15b (inhibitor) effectively diminished miR-15b expression (Fig. 6A) and suppressed cell proliferation of HepG2 cells, compared with the control group (Fig. 6B).

Effect of the downregulation of miR-15b expression on apoptosis and caspase-3 in the HepG2 cells. Next, we analyzed the effect of the downregulation of miR-15b expression on apoptosis and caspase-3 in the HepG2 cells. As expected, HepG2 cells transfected with the miR-15b inhibitor displayed an enhanced apoptosis rate (Fig. 7A) and caspase-3 activity (Fig. 7B), compared with the control group.

Effect of the downregulation of miR-15b expression on TGF- $\beta$ in the Hep 2 cells. We then assessed the relationship of miR-15b and TGF- $\beta$ signaling in the HepG2 cells. Downregulation of miR-15b expression reduced TGF- $\beta$ signaling in the HepG2 cells compared with the control group (Fig. 8).

Effect of the downregulation of miR-15b expression on T $\beta R I$ in the HepG2 cells. To reveal the effect of the downregulation of miR-15B on T $\beta R I$ in the HepG2 cells, T $\beta R I$ protein expression was assessed using western blot analysis. The protein expres- 

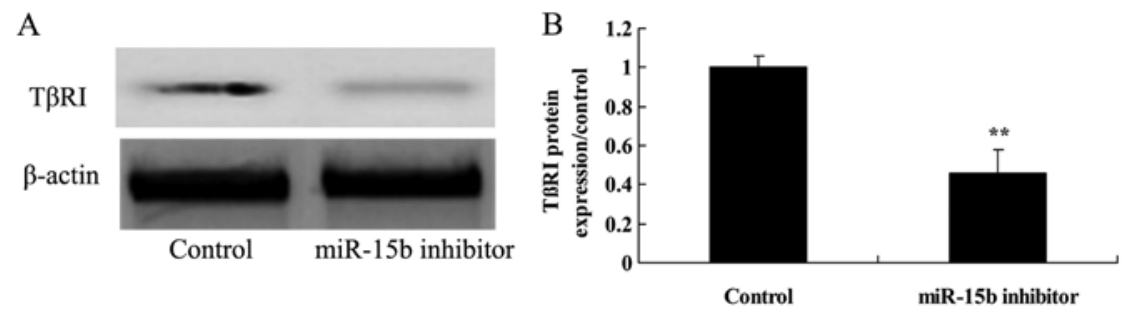

Figure 9. Effect of the downregulation of miR-15b expression on T $\beta R I$ in the HepG2 cells. Effect of the downregulation of miR-15b expression on T $\beta R I$ protein expression using Western blot (A) and T $\beta R I$ protein expression using statistical analysis (B) in the HepG2 cells compared with the control group. ${ }^{* * *} \mathrm{P}<0.01$ compared with the control group.
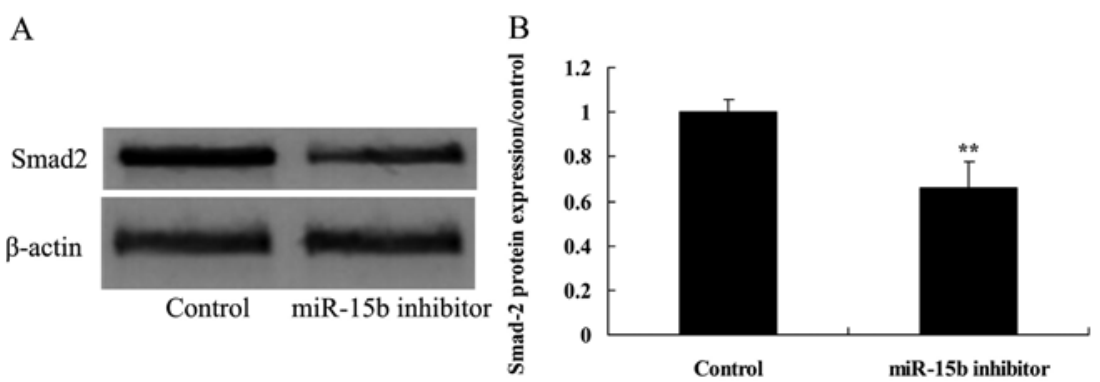

Figure 10. Effect of the downregulation of miR-15b expression on Smad2 in the HepG2 cells. Effect of the downregulation of miR-15b expression on Smad2 protein expression using Western blot (A) and Smad2 protein expression using statistical analysis (B) in the HepG2 cells. ${ }^{* *} \mathrm{P}<0.01$ compared with the control group.

A

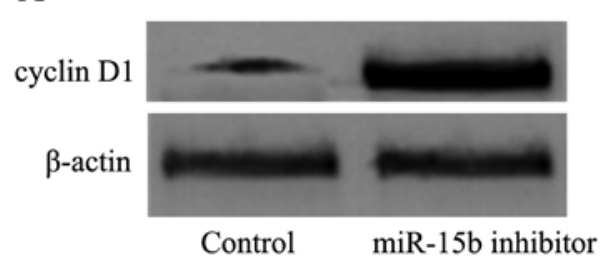

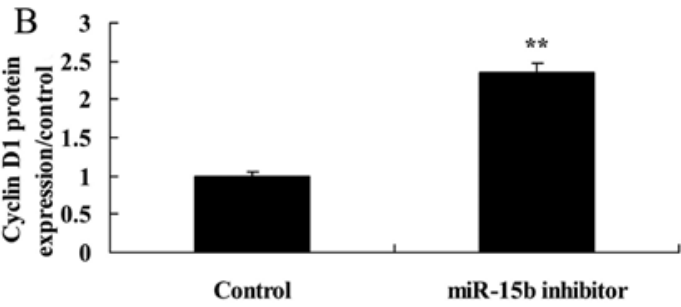

Figure 11. Effect of the downregulation of miR-15b expression on cyclin D1 in the HepG2 cells. Effect of the downregulation of miR-15b expression on cyclin D1 protein expression using Western blot (A) and D1 protein expression using statistical analysis (B) in the HepG2 cells. ${ }^{* *} \mathrm{P}<0.01$ compared with the control group.

sion of T $\beta R$ I was effectively inhibited by the downregulation of miR-15b, compared with the control group (Fig. 9).

Effect of the downregulation of miR-15b expression on Smad2 in the HepG2 cells. To further understand the functional effect of Smad2 in the HepG2 cells transfected by anti-miR-15b, Smad 2 protein expression was determined using western blot analysis. The results of the western blot analysis demonstrated that downregulation of miR-15b observably suppressed the protein expression of Smad2 in the HepG2 cells, compared with the control group (Fig. 10).

Effect of the downregulation of miR-15b expression on the level of cyclin D1 in the HepG2 cells. Next, we assessed the effect of the downregulation of miR-15b expression on cyclin D1 in the HepG2 cells. Cyclin D1 protein expression was detected using western blot analysis. As shown in Fig. 11, cyclin D1 protein expression was markedly enhanced by the downregulation of the expression of miR-15b, compared with the control group.

Effect of the downregulation of miR-15b expression on the level of Bax in the HepG2 cells. To this end, we co-transfected HepG2 cells with anti-miR-15b, and the protein expression of Bax was analyzed using western blot analysis. Bax protein expression was significantly activated by the downregulation of the expression of miR-15b, compared with the control group (Fig. 12).

\section{Discussion}

Identification of the risk factors of post-operative recurrence for HCC are an urgent quest. Research suggests that these high risk factors involve many aspects, such as surgical techniques, HCC background, tumor characteristics and perioperative 
A

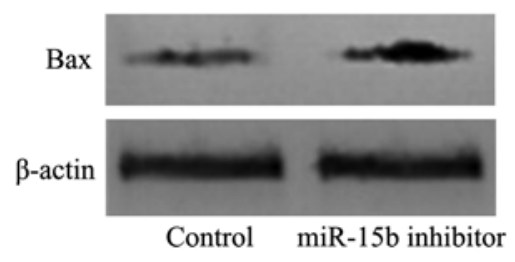

B

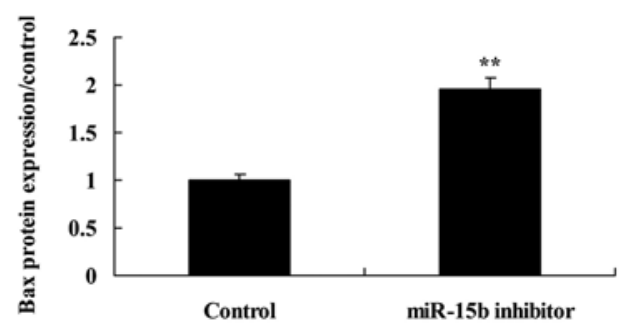

Figure 12. Effect of the downregulation of miR-15b expression on Bax in the HepG2 cells. Effect of the downregulation of miR-15b expression on Bax protein expression using Western blot (A) and Bax protein expression using statistical analysis (B) in the HepG2 cells. ** P<0.01 compared with the control group.

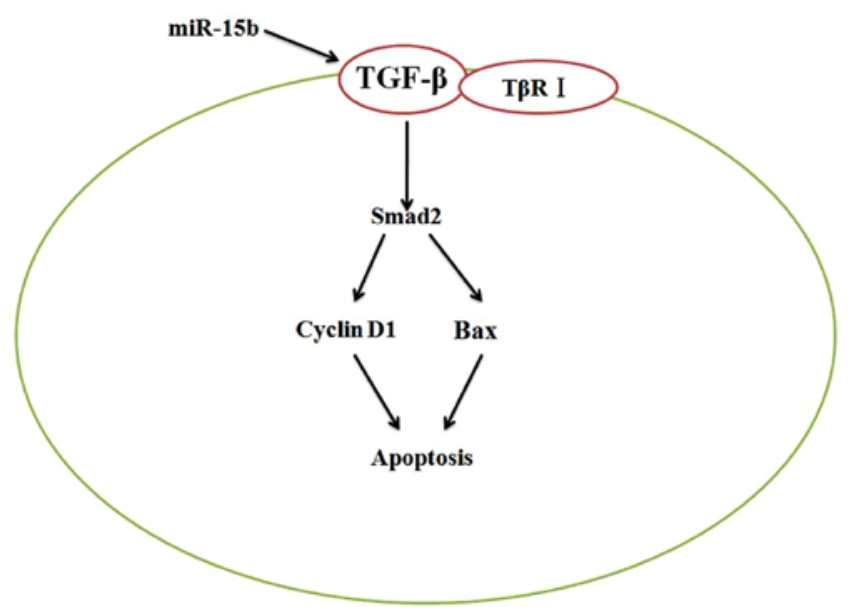

Figure 13. High expression of miR-15b predicts the poor prognosis of hepatocellular carcinoma patients after curative hepatectomy through TGF- $\beta$ / T $\beta$ RI-Smad2-cyclin D1/Bax.

treatment (1). These factors are reciprocal and synergistic. It is important for strict monitoring to detect postoperative recurrence (2). However, it is obviously unsuitable from the perspective of health economics to provide all patients with frequent comprehensive examinations. Consequently, it is essential for hepatic surgeons to properly control the factors associated with postoperative recurrence and identify highrisk populations to carry out suitable reexaminations and conduct subsequent adjuvant therapy (3). Here our results suggest that miR-15b promoted the proliferation of HCC cells, and downregulation of the expression of miR-15b suppressed cell growth and induced the apoptosis of HepG2 cells.

In the liver, TGF- $\beta$ can be secreted by various types of cells, including parenchymal and mesenchymal cells, such as endothelial cells, hepatic cells and HSCs (4). During developmental processes of hepatic fibrosis, TGF- $\beta$ can activate fibrotic cytokines and promote HSCs to differentiate into myofibroblasts (5). Continuous stimulation of TGF- $\beta$ can result in the accumulation of ECM and the synthesis of tissue inhibitor of metalloproteinases facilitating the production of hepatic fibrosis (6). In the present study, we showed that downregulation of the expression of miR-15b suppressed the growth of HCC HepG 2 cells through the TGF- $\beta$ signal. Furthermore, recent reports have identified that the microRNA-15 family inhibits the TGF $\beta$ pathway in the heart (7).
Smad protein molecules inhibit hepatic fibrosis by inhibiting the formation, phosphorylation and nuclear translocation of Smad protein molecules (8). After injection of adenovirus vector with $\mathrm{Smad} 7 \mathrm{cDNA}$ to rats, rat collagen contents were found to be reduced while the activation of HSCs was inhibited (8). Furthermore, in an in vitro experiment it was found that Smad7 influenced hepatic fibrosis through inhibition of phosphorylation of Smad2/3 and nuclear translocation of activated Smad (9). Hepatic fibrosis can be inhibited by reducing the contents of Smad3 and Smad4 or decreasing their activities. Importantly, our data suggest that downregulation of the expression of miR-15b observably suppressed Smad2 signaling in the HepG2 cells.

Smad7 could freeze activated Smad1 and Smad2 to degrade them. Therefore, ectopic expression of Smad 2 decreases expression of Smad1 and Smad2 while expression of Smad3 cannot be decreased (10). Smads2 is increased in the livers of rats with fibrosis while it was decreased in cirrhotic rats and humans (11). Overexpressed Smad2 reduces the production of HSC collagen. In humans, overexpression of Smad2 decreased levels of T $\beta R I$ and Smad7 (12). Meanwhile, levels of ECM and laminin were decreased. Stimulated by TGF- $\beta$, Smad 2 recruits $S m a d 7$ to form compounds in the cell nucleus (9). Under stimulation of TGF- $\beta$, compounds are transferred into the cytoplasm. Subsequently, these compounds interact with T $\beta$ RI. Our observations revealed that downregulation of the expression of miR-15b observably inhibited TGF- $\beta$ protein expression in the HepG 2 cells through T $\beta$ RI signal. Zhang et al demonstrated that miR-15b promotes epithelial-mesenchymal transition of pancreatic cancer through TGF- $\beta / T \beta R$ I signaling (13)

The Bcl-2 family are apoptosis factors and Bcl-2 is the strongest anti-apoptotic factor which is closely associated with the incidence and tolerance of tumors (14). Bcl-2 and Bax are antagonists and function in the form of a dipolymer. If expression levels are balanced, the cell lifespan is normal (15). When the quantity of Bcl-2 is relatively high, the heterodimer Bcl-2/ Bax is formed. When the quantity of Bax is relatively high, the homodimer Bax/Bax is formed, which promotes apoptosis by inhibiting the anti-apoptotic functions of Bcl-2 $(16,17)$. Therefore, it is generally thought that Bcl-2 and Bax play a fundamental role in cell apoptosis. Caspases directly participate in the early initiation of apoptosis and the transmission of apoptotic signaling while at the advanced stage they produce apoptosis characteristics such as shrinkage, nuclear division and DNA fracture (18). Caspases are located at the central position of the apoptosis process. Caspase- 3 is the key 
administrator of apoptosis (19). It is clear from our data, that downregulation of the expression of miR-15b significantly activated Bax protein expression and caspase- 3 activity in the HepG2 cells. Liu et al found that knockdown of NDRG2 sensitized cervical cancer HeLa cells through miR-15b and miR-16 expression mediated by Bcl-2 and Bax (17).

Taken together, our results demonstrate that miR-15b plays a role in the poor prognosis of HCC patients after curative hepatectomy. This outcome suggests that miR-15b is a potential therapeutic target for HCC. Our results showed that miR-15b mediates cell growth and induces apoptosis of liver cancer, possibly by directly targeting the TGF- $\beta$ /T $\beta$ RI-Smad2-cyclin D1/Bax signaling pathway (Fig. 13). Collectively, these data suggest that miR-15b and the TGF- $\beta$ /T $\beta$ RI-Smad2-cyclin D1/Bax signaling pathway may be potential therapeutic targets for HCC after curative hepatectomy and deserve further study.

\section{References}

1. El-Kady NM, Esmat G, Mahmoud EH, Darweesh SK, Mahmoud SH and Elagawy WA: Hypertonic saline-enhanced radiofrequency versus chemoembolization sequential radiofrequency in the treatment of large hepatocellular carcinoma. Eur J Gastroenterol Hepatol 25: 628-633, 2013.

2. Fernandez-Banet J, Lee NP, Chan KT, Gao H, Liu X, Sung WK, Tan W, Fan ST, Poon RT, Li S, et al: Decoding complex patterns of genomic rearrangement in hepatocellular carcinoma. Genomics 103: 189-203, 2014.

3. Sarwar S, Khan AA and Tarique S: Validity of alpha fetoprotein for diagnosis of hepatocellular carcinoma in cirrhosis. J Coll Physicians Surg Pak 24: 18-22, 2014.

4. Jiang F, Wang X, Liu Q, Shen J, Li Z, Li Y and Zhang J: Inhibition of TGF- $\beta /$ SMAD3/NF- $\kappa$ B signaling by microRNA-491 is involved in arsenic trioxide-induced anti-angiogenesis in hepatocellular carcinoma cells. Toxicol Lett 231: 55-61, 2014.

5. Lou XL, Deng J, Deng H, Ting Y, Zhou L, Liu YH, Hu JP, Huang XF and Qi XQ: Aspirin inhibit platelet-induced epithelial-to-mesenchymal transition of circulating tumor cells (Review). Biomed Rep 2: 331-334, 2014.

6. Yang J, Zheng J, Wu L, Shi M, Zhang H, Wang X, Xia N, Wang D, Liu X, Yao L, et al: NDRG2 ameliorates hepatic fibrosis by inhibiting the TGF- $\beta 1 /$ Smad pathway and altering the MMP2/TIMP2 ratio in rats. PLoS One 6: e27710, 2011.
7. Tijsen AJ, van der Made I, van den Hoogenhof MM, Wijnen WJ, van Deel ED, de Groot NE, Alekseev S, Fluiter K, Schroen B, Goumans MJ, et al: The microRNA-15 family inhibits the TGF 3 -pathway in the heart. Cardiovasc Res 104: 61-71, 2014

8. Gal A, Sjöblom T, Fedorova L, Imreh S, Beug H and Moustakas A: Sustained TGF beta exposure suppresses Smad and non-Smad signalling in mammary epithelial cells, leading to EMT and inhibition of growth arrest and apoptosis. Oncogene 27: 1218-1230, 2008.

9. Chegini N, Luo X, Ding L and Ripley D: The expression of Smads and transforming growth factor beta receptors in leiomyoma and myometrium and the effect of gonadotropin releasing hormone analogue therapy. Mol Cell Endocrinol 209: 9-16, 2003.

10. Jeong MH, Kim SJ, Kang H, Park KW, Park WJ, Yang SY and Yang DK: Cucurbitacin I attenuates cardiomyocyte hypertrophy via inhibition of connective tissue growth factor (CCN2) and TGF- $\beta$ /Smads signalings. PLoS One 10: e0136236, 2015.

11. Choi K, Lee K, Ryu SW, Im M, Kook KH and Choi C: Pirfenidone inhibits transforming growth factor- $\beta 1$-induced fibrogenesis by blocking nuclear translocation of Smads in human retinal pigment epithelial cell line ARPE-19. Mol Vis 18: 1010-1020, 2012.

12. Zheng F, Lu W, Wu F, Li H, Hu X and Zhang F: Recombinant decorin ameliorates the pulmonary structure alterations by down-regulating transforming growth factor-beta1/SMADS signaling in the diabetic rats. Endocr Res 35: 35-49, 2010.

13. Zhang WL, Zhang JH, Wu XZ, Yan T and Lv W: miR-15b promotes epithelial-mesenchymal transition by inhibiting SMURF2 in pancreatic cancer. Int J Oncol 47: 1043-1053, 2015.

14. Li CL, Chang L, Guo L, Zhao D, Liu HB, Wang QS, Zhang P, Du WZ, Liu X, Zhang HT, et al: $\beta$-elemene induces caspase-dependent apoptosis in human glioma cells in vitro through the upregulation of Bax and Fas/ FasL and downregulation of Bcl-2. Asian Pac J Cancer Prev 15: 10407-10412, 2014.

15. Reed JC: Proapoptotic multidomain Bcl-2/Bax-family proteins: Mechanisms, physiological roles, and therapeutic opportunities. Cell Death Differ 13: 1378-1386, 2006.

16. Cory S and Adams JM: Killing cancer cells by flipping the Bcl-2/Bax switch. Cancer Cell 8: 5-6, 2005.

17. Liu J, Yang L, Zhang J, Zhang J, Chen Y, Li K, Li Y, Li Y, Yao L and Guo G: Knock-down of NDRG2 sensitizes cervical cancer Hela cells to cisplatin through suppressing Bcl-2 expression. BMC Cancer 12: 370, 2012.

18. Ryoo HD and Bergmann A: The role of apoptosis-induced proliferation for regeneration and cancer. Cold Spring Harb Perspect Biol 4: a008797, 2012.

19. Chen H, Yang X, Feng Z, Tang R, Ren F, Wei K and Chen G: Prognostic value of caspase- 3 expression in cancers of digestive tract: A meta-analysis and systematic review. Int J Clin Exp Med 8: 10225-10234, 2015. 